\title{
Efficient 3D charge transport in planar triazatruxene-based dumbbell-shaped molecules forming a bridged columnar phase
}

Received 00th January 20xx, Accepted 00th January 20xx DOI: $10.1039 / x 0 x \times 00000 x$

\author{
Jiang Jing, ${ }^{a}$ Benoît Heinrich, ${ }^{\mathrm{b}}$ Alexis Prel, ${ }^{\mathrm{a}}$ Emilie Steveler, ${ }^{\mathrm{a}}$ Tianyan Han, ${ }^{\mathrm{a}}$ Ibrahim Bulut, ${ }^{\mathrm{c}}$ Stéphane \\ Méry, ${ }^{\mathrm{b}}$ Yann Leroy, ${ }^{\mathrm{a}}$ Nicolas Leclerc, ${ }^{\mathrm{c}}$ Patrick Lévêque, ${ }^{\mathrm{a}}$ Martin Rosenthal, ${ }^{\mathrm{d}}$ Dimitri A. Ivanove,f and \\ Thomas Heiser*a
}

\begin{abstract}
Planar conjugated molecular backbones are essential for achieving high charge carrier mobilities along molecular $\pi$-stacking directions but are often concomitant with poor charge transport in other directions. This is particularly the case for molecules that are functionalized with alkyl chains, which ensure good processability in solution but introduce detrimental insulating regions. Here, we show that soluble planar dumbbell-shaped molecules, composed of two triazatruxene (TAT) units covalently bonded to a central thiophene-thienopyrroledione-thiophene (TPD) segment self-assemble into an original structure that allows efficient 3D charge transport. Grazing-incidence wide-angle X-ray scattering investigations as well as micro-focus X-ray experiments on single crystals reveal that the TAT derivatives form a columnar-nematic mesophase in which columns of stacked TAT units spaced by molten chains are interconnected by TPD bridges. Upon annealing, a crystalline phase, stemming from the parent hexagonal mesophase, is obtained with the molecular stacking direction lying in-plane. Transport measurements in the crystalline phase reveal an unusually high out-of-plane hole mobility of $0.17 \mathrm{~cm}^{2} \mathrm{~V}$ ${ }^{1} \mathrm{~s}^{-1}$ and a lower limit for the in-plane mobility of $0.05 \mathrm{~cm}^{2} \mathrm{~V}^{-1} \mathrm{~s}^{-1}$. The results suggest that the TPD segments bridging neighboring stacks of TAT columns are responsible for efficient hole transport in 3D.
\end{abstract}

\section{Introduction}

Charge transport is known to be a limiting factor in the performance of organic electronic and photovoltaic devices. Much efforts have therefore been devoted to better understand the relationships between molecular structure, selfassembling and charge transport and to develop materials with high charge carrier mobilities. ${ }^{[1-6]}$ As strong intermolecular coupling and high structural order are recognized prerequisites for efficient charge transport, ${ }^{[7,8]}$ many planar conjugated units such as triphenylene, ${ }^{[9]}$ dinaphtho-thieno-thiophene (DNTT), ${ }^{[10,}$ 11] [1]benzothieno[3,2-b] benzothiophene (BTBT) derivatives, ${ }^{[12]}$ polycyclic aromatic hydrocarbons (PAHs), ${ }^{[13]}$ or triazatruxene $(\mathrm{TAT})^{[14,15]}$ have been used as molecular building blocks ${ }^{[16-18]}$

\footnotetext{
a. ICube Research Institute, Université de Strasbourg, CNRS, UMR 7357, 23 rue du Loess, 67037 Strasbourg, France.E-mail: thomas.heiser@unistra.fr

b. Institut de Physique et Chimie des Matériaux de Strasbourg, Université de Strasbourg, CNRS, UMR 7504, 23 rue du Loess, 67034 Strasbourg, France. . Institut de Chimie et Procédés pour l'Energie, l'Environnement et la Santé Université de Strasbourg, CNRS, UMR 7515, 25 rue Becquerel, 67087 Strasbourg, France.

d. European Synchrotron Radiation Facility (ESRF), Avenue des Martyrs 71, 38000 Grenoble, France.

e. Institut de Sciences des Matériaux de Mulhouse, IS2M, CNRS UMR7361, 15 Jean Starcky, Mulhouse 68057, France.

f. Faculty of Chemistry, Lomonosov Moscow State University, GSP-1, 1-3 Leninskiye Gory, 119991 Moscow, Russia.

+ Footnotes relating to the title and/or authors should appear here.

Electronic Supplementary Information (ESI) available: [details of any supplementary information available should be included here]. See DOI: 10.1039/x0xx00000x
}

and led to some of the highest mobilities reported so far for molecular semiconductors. However, beyond molecular planarity, charge transport anisotropy is another critical parameter that must be taken into account when seeking to improve charge transport. Indeed, S. Fratini et al. have recently shown that the degree of charge transport anisotropy determines the impact of dynamic disorder on charge carrier mobility. ${ }^{[1,19]}$ Their results imply that molecular packings leading to isotropic transfer integrals are more tolerant against disorder-induced carrier localization and are therefore more prompt to achieve high mobilities. In addition, isotropic charge transport also simplifies the elaboration of efficient organic devices by avoiding the requirement to align the high mobility axes with the charge flow direction (i.e. in-plane for field-effect transistors or out-of-plane for light emitting diodes or solar cells). Yet, with the exceptions of herringbone-type assemblies, common ordered molecular solid-state packings lead to intermolecular transfer integrals that are highly anisotropic. ${ }^{[1]}$ In the particular case of planar molecules that can be processed from solution, strong molecular $\pi-\pi$ stacking interactions often result in efficient charge transport along the molecular stacking, while the solubilizing alkyl chains hinder charge transfer in perpendicular directions. ${ }^{[20-22]}$ For instance, discotic molecules based on soluble TAT derivatives generally self-assemble into columnar structures that allow efficient carrier transport along the columnar direction only, giving rise essentially to onedimensional transport. ${ }^{[23]}$ Designing soluble planar molecular semiconductors that lead to strong $\pi$-stacking interactions while allowing efficient 3D charge transport is thus still a 
challenging and important target for organic electronics. In this context, the recent synthesis of a new family of donor (D)acceptor (A)-donor (D) type molecules based on two TAT electron donor units and a central thiophenethienopyrroledione-bithiophene (TPD) unit, reported by Han et al., ${ }^{[24]}$ is of particular interest. Indeed, one of these DAD compounds was found to support 3D charge transport despite the self-assembling into a columnar mesophase similar to those of other discotic TAT derivatives. ${ }^{[15,21]}$ First evidence for efficient 3D transport was provided by the promising out-ofplane and in-plane hole mobilities, of the order of $10^{-3} \mathrm{~cm}^{2} \mathrm{~V}^{-1} \mathrm{~s}^{-1}$, measured in as-deposited films. ${ }^{[24]}$ The presence of two planar TAT units as molecular end-groups may possibly be at the origin of this astonishing property. However, a full investigation and optimization of the molecular self-assembly of this compound and their consequences on charge transport is still lacking.

In this study, we explored the self-assembly and charge transport properties of this TAT-based dumbbell-shaped derivative in greater depth in order to elucidate and take advantage of the unique structural properties of this molecule to achieve efficient 3D charge transport. The out-of-plane hole mobility was measured in space-charge limited current (SCLC) devices and reached a maximum SCLC mobility of $0.17 \mathrm{~cm}^{2} \mathrm{~V}^{-1} \mathrm{~s}^{-}$ ${ }^{1}$ for the crystalline state, close to the highest values reported to date for soluble small molecules, ${ }^{[23]}$ which was measured along the molecular stacks in the columnar phase of a discotic TAT derivative. A lower limit of $0.05 \mathrm{~cm}^{2} \mathrm{~V}^{-1} \mathrm{~s}^{-1}$ was found for the in-plane hole mobility in field-effect transistors. Importantly, the structural investigations demonstrate that the efficient outof-plane carrier transport occurs along a direction perpendicular to the molecular stacking, in strong contrast with the properties of other TAT-based discotic molecular semiconductors. Overall, our results show that this remarkable behavior can be linked to the self-assembly of this TAT-based dumbbell-shaped derivative into an unusual and distinctive molecular network that comprises efficient transport pathways along three dimensions. Finally, although performances are still low compared to best liquid-crystalline 2D semiconductors ${ }^{[25]}$, the bridged columnar architecture represents a versatile alternative to other promising liquid-crystalline 3D structures such as bicontinuous cubic phases ${ }^{[26]}$.

\section{Results and discussion}

\subsection{Thermal properties and thin film solid states}

The chemical structure of the investigated TAT derivative,

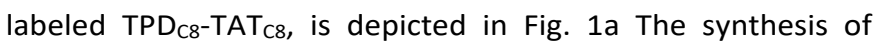
$\mathrm{TPD}_{\mathrm{C8}}-\mathrm{TAT}_{\mathrm{C} 8}$ and related differential scanning calorimetry (DSC) measurements have been reported previously. ${ }^{[24]}$ The DSC a)

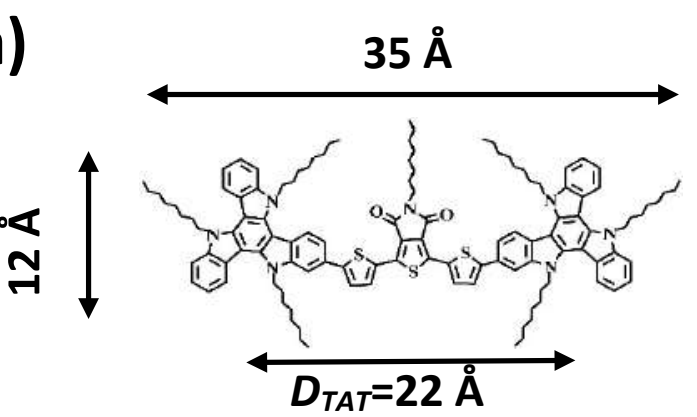

c)

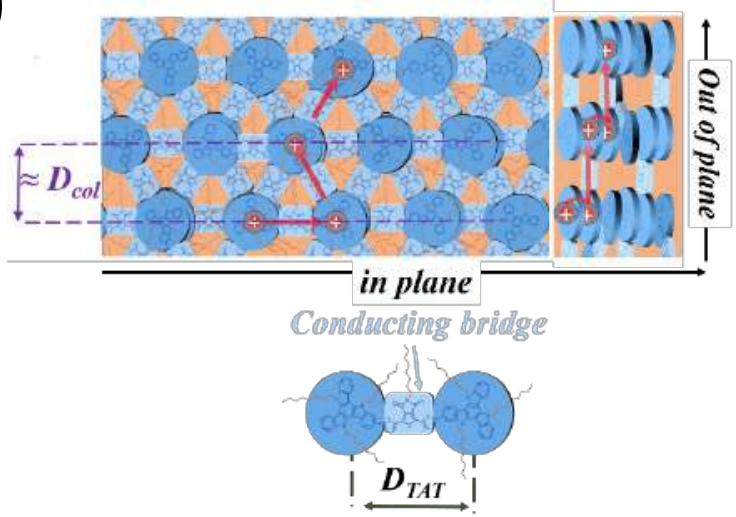

b)

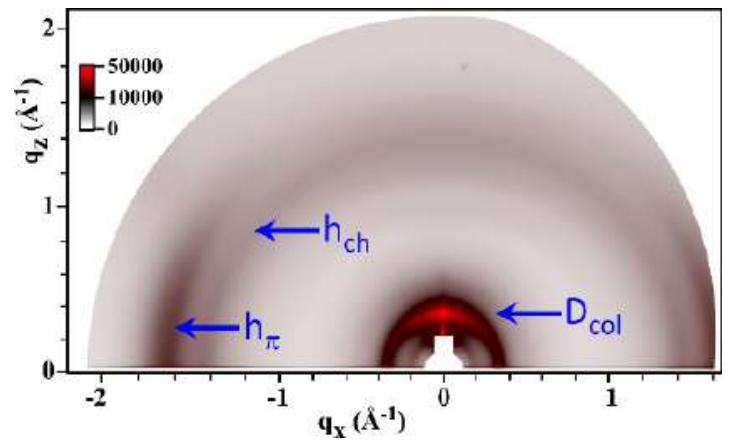

d)

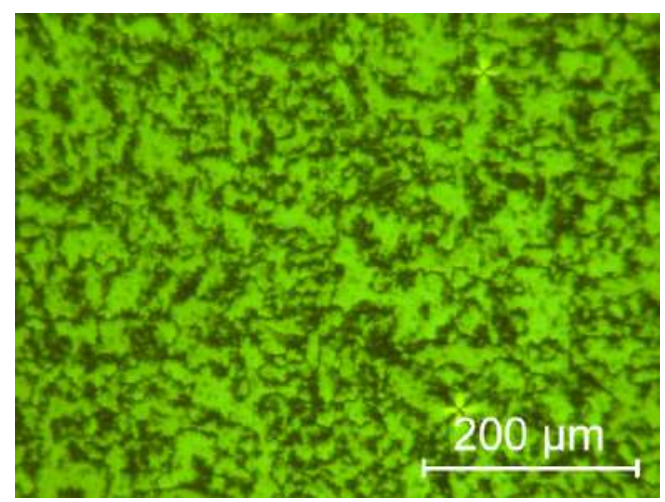

Fig. 1 (a) Chemical structure of a TPD $\mathrm{CB}_{\mathrm{C}} \mathrm{TAT}_{\mathrm{C} 8}$ molecule. (b) GIWAXS pattern of a columnar-nematic thin film on top of a silicon wafer. (c) Schematic view of the average local-range molecular packing of the columnar-nematic phase and illustration of charge transport pathways. The system is a fluid or frozen liquid crystal and involves a large amount of structural disorder which is illustrated in the right sketch. (d) Polarized optical micrograph of a columnar-nematic thin film. 
investigations (Fig. S1a) showed that the crude sample is in a mesomorphic solid state and transforms on heating to a columnar-nematic liquid crystal (LC) phase that freezes into a solid state on subsequent cooling. ${ }^{[21,24]}$ Crystallization from frozen mesophase never occurred, even after years' storage at room temperature. However, on staying in the nematic phase for a few minutes above $120^{\circ} \mathrm{C}$, nucleation of a crystalline phase is observed, giving rise to cold crystallization and melting peaks in heating DSC traces. The crystalline phase melts at $165^{\sim} 170{ }^{\circ} \mathrm{C}$, thus above the clearing temperature in the nematic state, which is therefore a monotropic phase in the sense that it can only be obtained on cooling from the isotropic liquid, but not on heating from the crystal.

The structure and morphology of $\mathrm{TPD}_{\mathrm{C} 8}-\mathrm{TAT}_{\mathrm{C} 8}$ thin films, on either silicon or glass/ITO substrates, were investigated by Grazing Incidence Wide-Angle X-ray Scattering (GIWAXS). Details on film deposition and thermal processing are given in section 3. The GIWAXS pattern obtained on as-deposited films displayed the characteristic broad scattering signals of the frozen columnar-nematic phase shown by bulk material (Fig. 1b). ${ }^{[24]}$ Specifically, these signals originate from $\pi$-stacking distances within columns of TAT units $\left(h_{\pi}=3.8 \AA\right.$ ) , packing distances between molten alkyl chains $\left(h_{c h}=4.5 \AA\right)$ and lateral spacing between columns $\left(D_{c o l}=18 \AA\right)$. Correlation lengths calculated from peak widths using Scherrer equation with shape factor $\mathrm{K}=0.9$ are about 3-3.5 $\mathrm{nm}$ for $h_{\pi}$ and $7 \mathrm{~nm}$ for $D_{\text {col, }}$, representing 8-9 molecules in the piling direction and 4 columns in the lateral direction (Fig.S2). While $h_{c h}$ and $D_{c o l}$ formed continuous rings, $h_{\pi}$ appeared as an arc centered on pattern equator, revealing that the direction of alignment of the columns (i.e. the nematic director) is parallel to the surface and randomly switches between nematic domains while keeping its in-plane orientation. The unicity of small-angle peak indicates a hexagonal-like local range arrangement with unique column row spacing given by peak position $D_{c o l}$ and distance between columns given by $(2 / \sqrt{ } 3) D_{\text {col }}{ }^{[27]}$. The close matching of this distance ( $21 \AA$ ) and of the distance separating the center of both TAT units on a given molecule ( $22 \AA$ ) indicates that both TAT units are involved into two neighboring columnar stacks. This generates an original columnar-nematic self-assembly in which the columns of stacked TAT units spaced by molten chains are interconnected by TPD bridges, as sketched in Fig. 1c. Although, for clarity, this scheme exaggerates the regularity and persistence length of structural order, it emphasizes that each molecule is involved in two neighboring TAT-based columns, introducing potentially efficient hole conducting pathways through the insulating aliphatic periphery of the columns and giving rise to what might be termed a "bridged columnar phase".

As with the bulk material, specific heating conditions in the range of the fluid nematic phase (see Experimental Section) initiates a crystallization process (Fig. 2). Accordingly, annealing as-deposited layers at $145^{\circ} \mathrm{C}$ causes the molecules to crystallize in the form of micrometre-sized highly birefringent needle-like crystals, seen in the polarized optical micrograph (Fig. 2a). The average size and density of crystalline needles depends on the spin-coated layer thickness and on the annealing process
(Fig.S13). While after 5 minutes annealing into the isotropic phase at $180^{\circ} \mathrm{C}$ and rapid cooling to room temperature smooth films of the material in its columnar nematic state are obtained (Fig. 1d), subsequent heating to $145^{\circ} \mathrm{C}$ leads to an assembly of larger but fewer crystals (Fig. 2b, Fig. S13 and Fig. S14), indicating that annealing into the isotropic phase reduces the number of nucleation centers. The crystal growth rate at $145^{\circ} \mathrm{C}$ was estimated to $25 \mu \mathrm{m} / \mathrm{h}$ (Fig. S13f).

The GIWAXS pattern of a crystallized film is henceforth composed of spot-like reflections due to the spontaneous orientation of crystallites with respect to surface (Fig. 2c). In the small-angle region, the $(h k 0)$ reflexes were sufficiently sharp for indexation according to an oblique lattice of parameters: $a_{2 \mathrm{D}}=$ $25.2 \pm 0.1 \AA, b_{2 \mathrm{D}}=25.7 \pm 0.1 \AA, \gamma_{2 \mathrm{D}}=131 \pm 1^{\circ}$, which corresponds to the lateral arrangement of piled molecules. The fact that both parameters of the unit cell are close to each other and that the angle is not too far from $120^{\circ}$, it can be supposed that the observed crystalline phase stems from the parent hexagonal liquid-crystalline phase. Crystalline domains are predominantly oriented with the $(a, b)$-plane orthogonal and $b$-axis parallel to substrate, implying that the directions of molecular piling lie in the film plane as for the initial mesomorphic structure. The grouping of strong reflections at low $q_{x}$ is consistent with this finding. Unfortunately, the diffraction peaks in the wide- and medium-angle range are broadened and cannot be resolved in individual reflections, which precludes the full determination of the three-dimensional structure at this stage. At the same time, all trials to grow crystals from solutions failed, presumably because a mesomorphic state was systematically obtained. It turned out that the crystalline state is currently only available through the above-described thermal treatment in form of microcrystals, which are unsuitable for laboratory XRD. However, it was possible to conduct complementary microfocus $\mathrm{X}$-ray experiments on individual microcrystals of a crystallized thin film using synchrotron radiation.

Spatially resolved X-ray diffractograms were obtained by performing 2D scans of large crystals with a step of $1 \mu \mathrm{m}$. The aim of the measurements was to identify the crystallographic direction corresponding to the long axis of the needle-like crystals such as the ones observed with an on-axis optical microscope installed at the ID13 beamline of the ESRF (Fig. S3). The micro-diffractogram acquired on a relatively isolated crystal shown in the magnified image in Fig. S3b, displays sharp reflexes typical of a crystalline phase (Fig. 2d), which is in line with the data from GIWAXS measurements. Similarly, the $d$-spacings of the $(h k l)$ reflexes can be extracted from the pseudo-powder 1Dreduced diffractogram and fitted to a 2D lattice with $a=$ $24.63 \pm 0.01 \AA, b=25.67 \pm 0.01 \AA$ and $\gamma=129.55 \pm 0.01^{\circ}$. Given that the sample-to-detector distance is precise within $20 \mu \mathrm{m}$ and that the measurements are done on isolated crystallites, these values are very accurate and specific of the monocrystalline structure, while the ones obtained from GIWAXS provide an average set of parameters for the whole crystallized film.

The crystallographic direction corresponding to the long axis of the crystal can be determined as follows. Firstly, the two strongest in-plane $(h k 0)$ reflections are indexed to $(\overline{3} 50)$ and 


\section{a)}

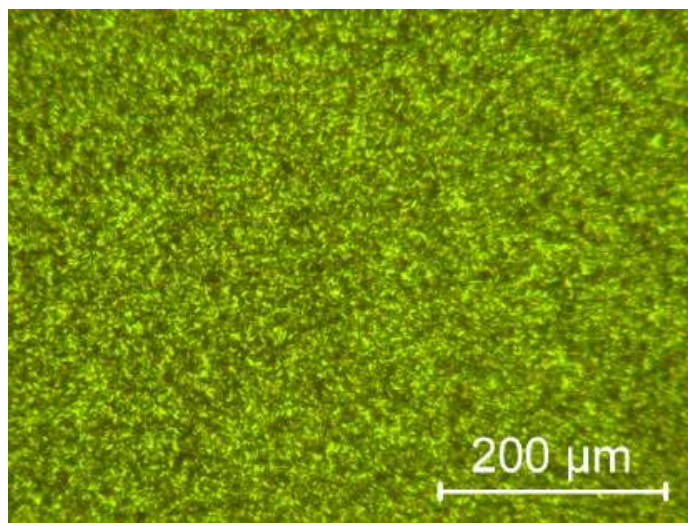

b)

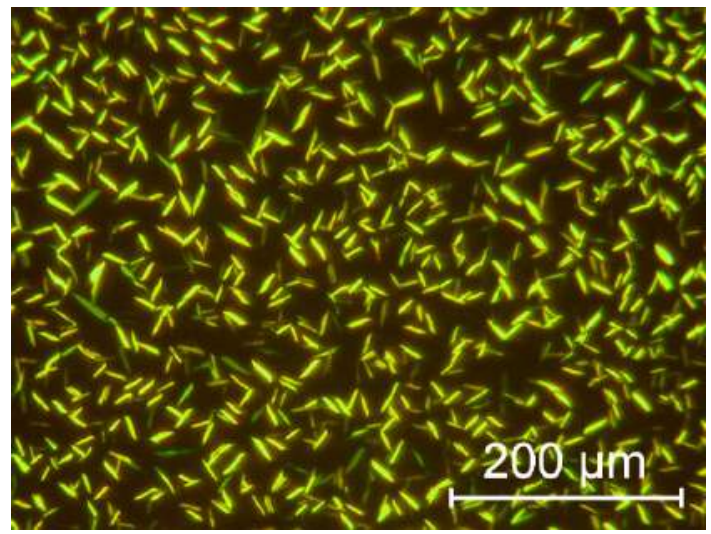

c)
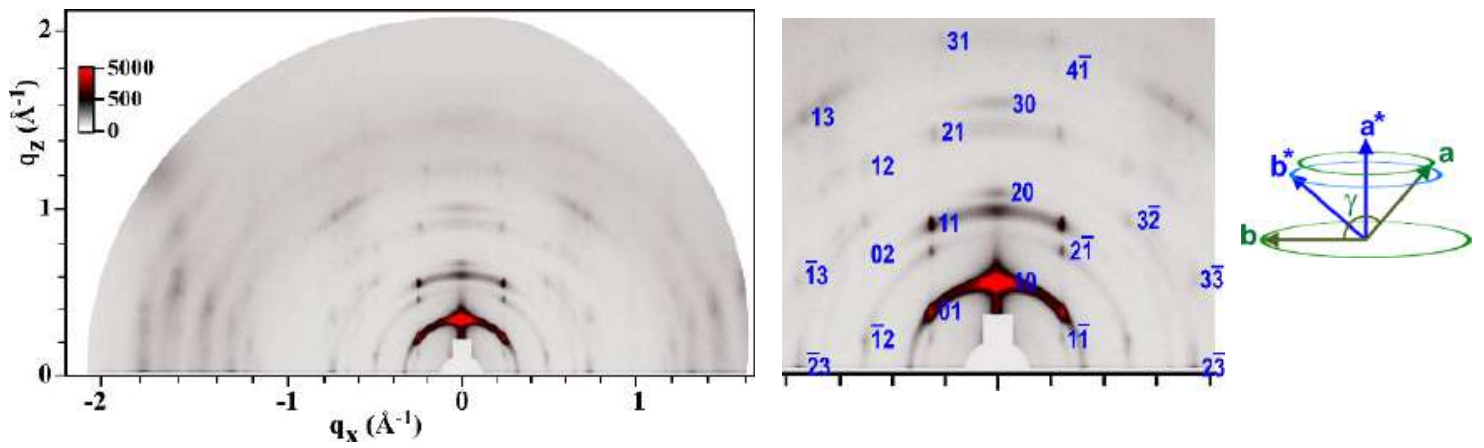

\section{d)}

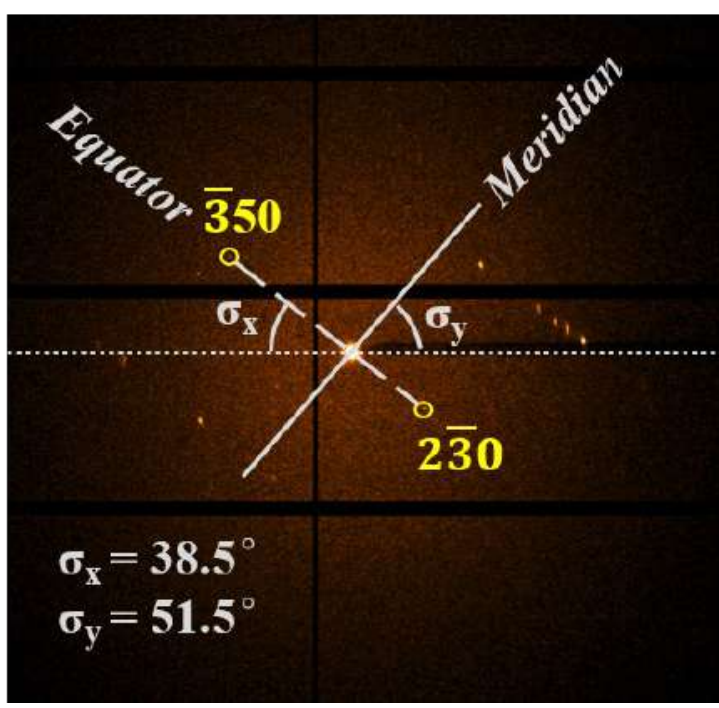

Fig. 2 (a) Polarized optical micrograph (POM) of a thin film of small crystals. (b) POM of a thin film composed of large crystals. (c) GIWAXS pattern of the crystalline thin film corresponding to (a), indexation of reflection spots (blue labels), and orientations of sublattice axes in reciprocal space (blue) and direct space (green). (d) Microfocus X-ray diffraction on an isolated crystal of a thin film similar to (b); $\overline{3} 50$ and $2 \overline{3} 0$ are the Miller indices of reflections lying on the equator of the pattern; $\sigma_{x}$ and $\sigma_{y}$ are the inclination angles of the meridian in the image (see also Fig. $\$ 3$ for comparison with crystal orientation). 
$(2 \overline{3} 0)$, the latter being also the strongest in-plane reflection in the GIWAXS pattern (Fig. 2c). The line connecting the (hkO) reflections is called equator of the pattern. It is observed that the perpendicular direction (i.e., meridian) is very close to the long axis of the crystal as can be seen from the inclination angles $\sigma_{y}$ and $\sigma_{x}$. Therefore, the long axis of the crystals corresponds to the direction of $c$-axis, which effectively lies in the film plane, as the direction of columns in the parent nematic phase.

For both columnar-nematic and crystalline solid states, the molecular piling direction is thus oriented parallel to the substrate and should support in-plane charge transport. On the other hand, for out-of-plane charge transport, the hopping pathways between adjacent molecular stacks are most important. In conventional molecular semiconductors that selfassemble into lamellar or columnar structures, the molecular stacks are generally separated by interlayers of alkyl chains that hinder charge transport perpendicular to the stacking direction. In contrast, for TPD $\mathrm{CB}_{\mathrm{C}} \mathrm{TAT}_{\mathrm{C} 8}$ in the columnar-nematic phase, the molecules bridging neighboring columns should support such a transport (as illustrated in Fig. 1c). The same likely holds for the crystalline films, with molecules bridging adjacent columns or layers through the insulating chain domains. However, since a full elucidation of the crystalline structure is not yet possible, measuring hole transport perpendicular to the substrate is currently the only way to probe the existence of such conducting inter-columnar pathways.

\subsection{Charge transport measurements}

Space-charge limited current devices provide a convenient and well-established way to study charge transport in semiconducting thin films perpendicular to the substrate and is also the method we have chosen to investigate out-of-plane charge transport in TPD ${ }_{C 8}-T_{A T}$ thin films..$^{[4,28-30]}$ As part of this work, two distinct hole-only SCLC device structures have been developed, referred to as "standard" and "thick" devices, respectively (Scheme S1 and S2). Standard devices were fabricated by sequential deposition of the hole injection and transporting layers and the organic semiconducting layer, the thickness $L$ of the latter being of the order of a few hundreds of nanometers.

Controlling the semiconductor thickness is crucially important for SCLC measurements, since the current density scales as $L^{-3}$. Surface roughness can lead to a significant overestimation of the hole mobility, as shown by numerical simulations in the supporting information (Fig. S4 and S5). Since crystalline $\mathrm{TPD}_{\mathrm{C8}}$ $\mathrm{TAT}_{\mathrm{C} 8}$ thin films exhibit a pronounced surface roughness (Fig. S1b and S1c), we developed the "thick" device structure to minimize the error margin on $L$. Thick devices consist of an assembly of two ITO-glass substrates coated with thin hole transporting layers and separated by micrometer-sized spacers (Scheme S2b). The interspace between both substrates is filled by a layer of $\mathrm{TPD}_{\mathrm{C8}}-\mathrm{TAT}_{\mathrm{C8}}$ powder. The sample is then annealed under mechanical pressure (applied to both substrates) to form either the crystalline or the nematic phase. In contrast to standard devices, the molecular layer thickness is in this case of the order of tens of micrometers and is well-defined even in the crystalline state. For both structures, the device fabrication and annealing conditions are described in more detail in section 3 .

The measurements were performed at room temperature in either the glassy nematic state or the crystalline state. Representative room-temperature current density-voltage curves measured in both solid states and both device architectures are plotted in Fig. 3. Additional experimental results are given in the supporting information. For each device structure, measurements on different film thicknesses have been performed to verify the absence of current-limiting injection barriers (Fig. S6 and S7). In accordance with MottGurney's law (see equation 1 below), the current densityvoltage $(J-V)$ curves measured for different $L$ overlap in the high voltage range when $J \times L$ is plotted versus $V / L$ (Fig. S6a and S7a). ${ }^{[31,32]}$ Interestingly, for thick devices, the curves coincide as well in the lower voltage range, when $J \times L$ is traced versus $V$ (Fig. $\mathrm{S} 6 \mathrm{~b})$, as predicted by Ohm's law, in contrast to standard devices (Fig. S7b). This can be understood by considering that in thick devices the background carrier concentration $\left(p_{o}\right)$ is fixed by residual non-intentional dopants and is independent of $L$, while in standard devices the average $p_{o}$ is determined by carrier indiffusion from contacts and decreases with increasing L. $^{[31]}$ Moreover, the ohmic resistance of thick devices (determined from the slope of the $J(V)$ curves at low voltages) varies linearly with $L$, as expected, and scales down to zero at zero layer thickness (Fig. S6c). The charge carrier injection barrier can therefore be considered to be negligible in these devices.

To estimate the hole mobility, we fitted the $J(V)$ curves to the following equation: ${ }^{[31-33]}$

$$
J=q \mu_{h} p_{o} \frac{V}{L}+\frac{9}{8} \varepsilon_{o} \varepsilon_{r} \mu_{h} \frac{V^{2}}{L^{3}}
$$

where the first term corresponds to Ohm's law and the second to Mott-Gurney's law. A field independent mobility $\left(\mu_{h}\right)$ and $p_{o}$ are used as fitting parameters. The dielectric constant $\left(\varepsilon_{r}\right)$ was estimated to 3.0 from the geometric capacitance of a standard SCLC device. The relevance to compare SCLC data to a sum of both laws has been recently discussed by Jason A. Röhr ${ }^{[33]}$ and was confirmed by our numerical simulations (Fig. S4).

The results obtained on standard devices agree well with equation (1) (Fig. 3a and 3b), suggesting that carrier transport in thin layers can be described by a field-independent hole mobility. The corresponding room temperature mobility data and $p_{o}$ values are summarized in Table 1 . The error margins are based on the histograms shown in Fig. S8 and correspond to the interval that includes $68 \%$ of the number of counts. In the columnar nematic phase, the SCLC mobility equals $(5.0 \pm 0.8) \times 10$ ${ }^{3} \mathrm{~cm}^{2} \mathrm{~V}^{-1} \mathrm{~s}^{-1}$, which is a factor of two above the previously reported value measured in as-deposited films. ${ }^{[2]}$ This difference is likely due to the larger columnar nematic domains and higher structural order after annealing. For the crystalline films, a remarkably high mobility of $0.34 \pm 0.20 \mathrm{~cm}^{2} \mathrm{~V}^{-1} \mathrm{~s}^{-1}$ is found 
a)

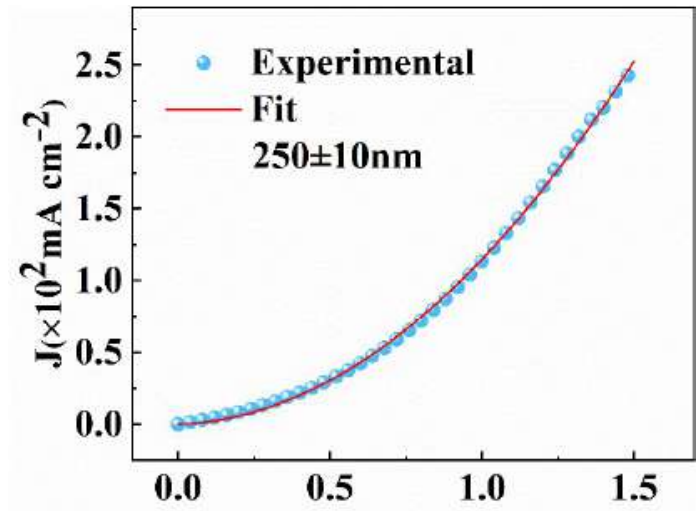

c)

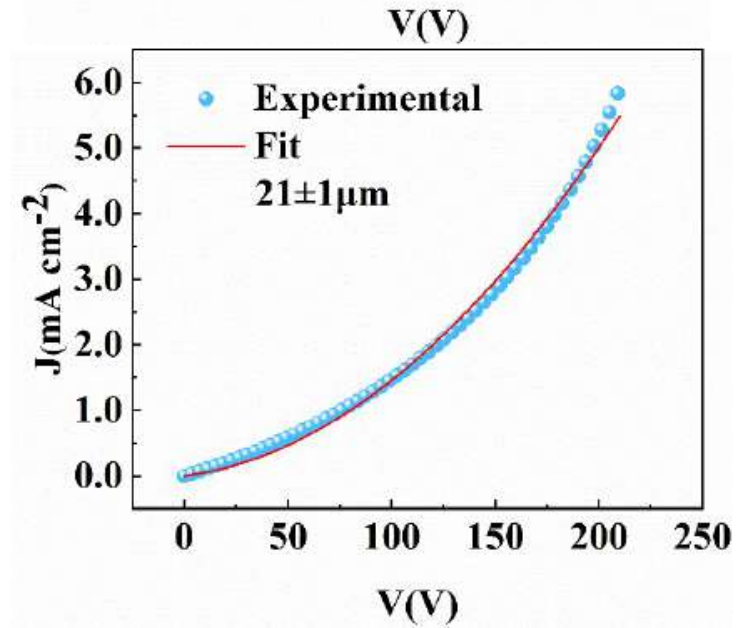

b)

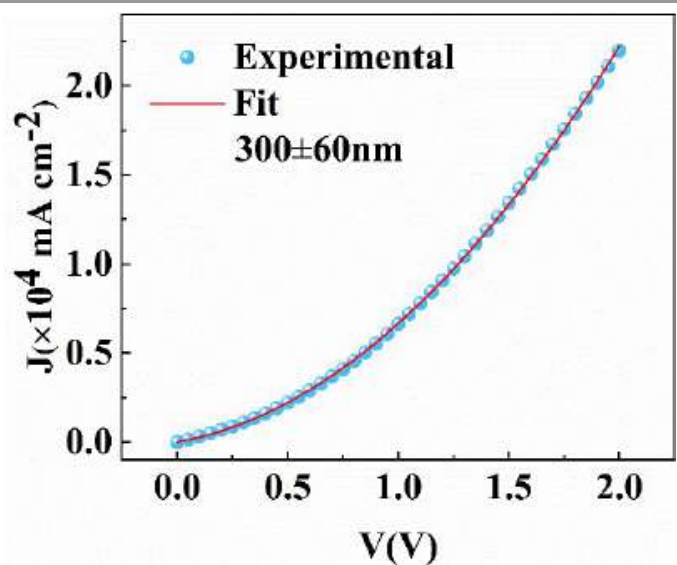

d)

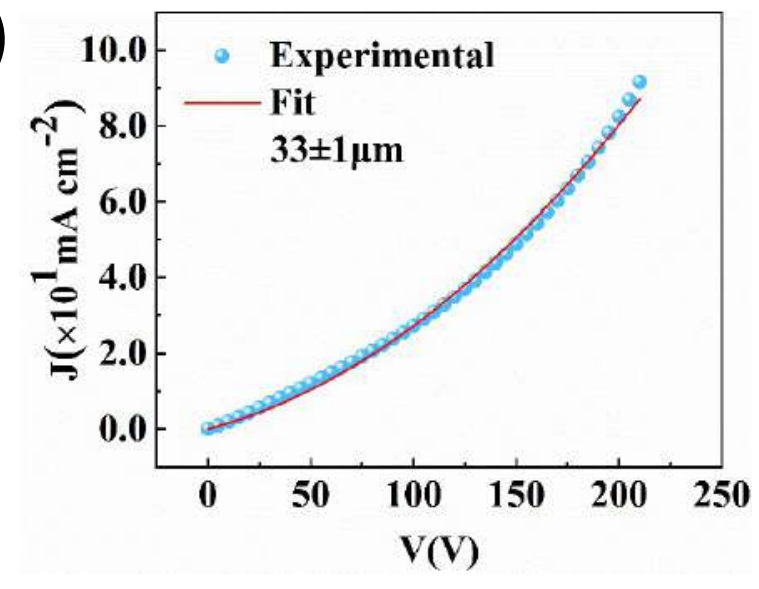

Fig. 3 Room temperature current-voltage characteristics of SCLC devices for different device structures and solid states. (a) Standard device in the columnar-nematic state $(250 \pm 10 \mathrm{~nm}$ film thickness). (b) Standard device in the crystalline state (300 $\mathrm{nm}$ average film thickness with a surface roughness of $60 \mathrm{~nm}$ ). (c) Thick device in the columnar-nematic state ( $21 \pm 1 \mu \mathrm{m}$ layer thickness). (d) Thick device in the crystalline state $(33 \pm 1 \mu \mathrm{m}$ layer thickness). The red solid lines represent fits to equation (1) (corresponding $\chi^{2}$ statistics: (a) 0.9993, (b) 0.9999, (c) 0.9949, (d) 0.9969)

Table 1. Charge transport properties of TPD $\mathrm{CB}_{-} \mathrm{TAT}_{\mathrm{C} 8}$ in SCLC and OFET devices

\begin{tabular}{ccccc}
\hline \multirow{2}{*}{ Solid state } & Devices & $\begin{array}{c}\mu_{h} \text { at } R T \\
{\left[\mathrm{~cm}^{2} \mathrm{~V}^{-1} \mathrm{~s}^{-1}\right]}\end{array}$ & $\begin{array}{c}p_{0} \text { at } R T \\
{\left[\mathrm{~cm}^{-3}\right]}\end{array}$ & $\begin{array}{c}E_{a} \\
{[\mathrm{meV}]}\end{array}$ \\
\hline \multirow{2}{*}{ Glassy nematic } & Standard SCLC & $(5.0 \pm 0.8) \times 10^{-3}$ & $(1.7 \pm 0.3) \times 10^{14}$ & $120 \pm 10$ \\
& Thick SCLC & $(2.7 \pm 0.5) \times 10^{-3}$ & $(3.3 \pm 0.7) \times 10^{13}$ & - \\
\cline { 2 - 5 } Crystalline & Top contact OFET & $(2.3 \pm 0.7) \times 10^{-4}$ & - & $30 \pm 8$ \\
& Standard SCLC & $* 0.34 \pm 0.20$ & $(1.6 \pm 1.2) \times 10^{15}$ & - \\
& Thick SCLC & $0.17 \pm 0.05$ & $(2.6 \pm 2.0) \times 10^{13}$ & - \\
& Top contact OFET & $(3.0 \pm 1.0) \times 10^{-3}$ & & - \\
\hline
\end{tabular}


when using the average thickness $\langle L>$. However, this value is likely to be overestimated due to the high surface roughness, as shown by the simulated current-voltage characteristics for a rough surface profile (Fig. S5).

The results obtained on thick devices, for both solid states, follow closely, but not fully, equation (1) (Fig. 3c and 3d). The slight deviation may be the consequence of an electric field dependent hole mobility. $\left.{ }^{[4}, 34,35\right]$ Although the origin and amplitude of the latter remain uncertain, we tentatively attribute its existence to the larger number of disordered domains in thick devices (i.e. crystal or domain boundaries). In the following, this rather minor effect will be neglected. The corresponding effective field independent mobility values are summarized in Table 1 . In the nematic state, the mobility is found to be $(2.7 \pm 0.5) \times 10^{-3} \mathrm{~cm}^{2} \mathrm{~V}^{-1} \mathrm{~s}^{-1}$, a value slightly below the mobility measured in standard devices. Since $p_{o}$ is also lower by roughly one order of magnitude, it is possible that the lower mobility is the outcome of a reduced occupancy rate of deep electronic states, which may be acting as hole traps. ${ }^{\left[{ }^{[6-38]}\right.}$ It is worth to note that the comparable mobility values observed in the standard and thick SCLC devices suggest that the TAT columns are similarly oriented (i.e. in plane) in both cases. The bright Schlieren textures observed by POM on thick SCLC devices confirm this assertion (Fig. S2c). In the case of crystalline films, the field independent mobility value is estimated to $0.17 \pm 0.05 \mathrm{~cm}^{2} \mathrm{~V}^{-1} \mathrm{~s}^{-1}$. Although lower than the value measured in standard devices, this value is close to the highest SCLC mobility reported so far for solution-processed molecular semiconductors. ${ }^{[13,23,39,40]}$ The factor of two difference in mobility may be the consequence of both, the higher $p_{o}$ and the higher surface roughness of standard devices.

We further evaluated the quality of charge transport in both solid states by measuring the SCLC mobility as a function of temperature (Fig. S9). Only the temperature dependent response of standard devices is reported, since charge transport should be less impacted by crystal boundaries in these devices and since the surface roughness should not interfere with the temperature dependency of the current. Given the rather narrow temperature range accessible to our equipment, we fitted our data to a simple Arrhenius law defined by:

$$
\mu_{h}=\mu_{\infty} \cdot \exp \left(-\frac{E_{a}}{k T}\right)
$$

where $E_{a}$ is the activation energy for charge carrier hopping, $k$ the Boltzmann constant, $T$ the absolute temperature and $\mu_{\infty}$ a pre-factor (Fig. S9b). In the nematic state, $E_{a}$ is estimated to $0.12 \pm 0.01 \mathrm{eV}$, which is near the lower limit of the range generally reported for organic semiconductors (0.1 0.6) eV..$^{[41-}$ ${ }^{43]}$ For crystalline films on the other hand, the activation energy drops down to $0.030 \pm 0.008 \mathrm{eV}$, which is exceptionally low and corroborates the existence of highly efficient pathways for charge transport perpendicular to the molecular stacking direction
We finally assessed in-plane charge transport in both, nematic and crystalline states, by measuring the hole mobility in topcontact-bottom-gate organic field-effect transistors (OFET) with channel widths varying between 60 and $90 \mu \mathrm{m}$. For the nematic state, the as-deposited film was annealed for 5 seconds into the isotropic phase $\left(180{ }^{\circ} \mathrm{C}\right)$ followed by natural cooling to room temperature, while for the crystalline state, the film was annealed at $145{ }^{\circ} \mathrm{C}$ for 40 minutes to obtain a uniform distribution of relatively small crystalline needles (Fig. S10a). More details on the OFET preparation conditions, device geometry as well as the measured electrical output and transfer characteristics are displayed in the supporting information (Scheme S3 and Fig. S10). For the nematic state, the field-effect mobility was estimated from the saturation regime to $2 \times 10^{-4}$ $\mathrm{cm}^{2} \mathrm{~V}^{-1} \mathrm{~s}^{-1}$. This value is about one order of magnitude lower than that of the SCLC mobility, despite the fact that charge transport should occur preferentially along the molecular stacking direction, which lies in-plane in OFET and SCLC devices. Possible reasons for this rather moderate value could be the small columnar correlation length, but also, and most importantly, the fact that since nematic domains are distributed randomly in-plane, not all of them are best oriented for an efficient conduction through the channel length. On the other hand, random in-plane orientation should not affect charge transport in SCLC devices.

For the crystalline state, high contact resistances led unfortunately to strongly non-linear transfer characteristics and allowed only a rough estimate of the field-effect mobility in the saturation regime. The latter was found to be approximately $3 \times 10^{-3} \mathrm{~cm}^{2} \mathrm{~V}^{-1} \mathrm{~s}^{-1}$ (Table 1 ). As this value is likely to be impacted by non-negligible charge carrier trapping, inter-crystal charge transport (the average crystal size being smaller than the channel length) and contact resistance, they represent a lower limit for the hole mobility. To minimize the impact of intercrystal charge carrier hopping on the mobility value, we investigated additional bottom-contact-bottom-gate transistors elaborated with large crystals and using a channel length $(2.5 \mu \mathrm{m})$ that was smaller than the average crystal size (Fig. 4 and S11). In these devices, a majority of molecular crystals bridged the gap between source and drain electrodes, covering a significant fraction of the channel width $(W)$. By considering an effective channel width that was determined optically to $0.17 \pm 0.02 \mathrm{~cm}$, (Fig. S11), we estimated the fieldeffect mobility in the saturation regime to $0.050 \pm 0.010 \mathrm{~cm}^{2} \mathrm{~V}^{-1} \mathrm{~s}^{-}$ ${ }^{1}$ (Table 1). Although this value remains underestimated due to the pronounced contact resistance (Fig. $4 \mathrm{c}$ and $4 \mathrm{~d}$ ), it is more than one order of magnitude above the value measured in large-channel top-contact OFETs, pointing out that charge transport may occur very efficiently also along the long axis of the crystalline needles, i.e. along the direction of molecular stacking. More advanced device structures and further optimization of the crystal growth, which are required to assess the in-plane mobility more accurately, lies beyond the scope of the present article.

Overall, the mobility data extracted from SCLC and OFET measurements, summarized in Table 1, point out a one order of 
a)

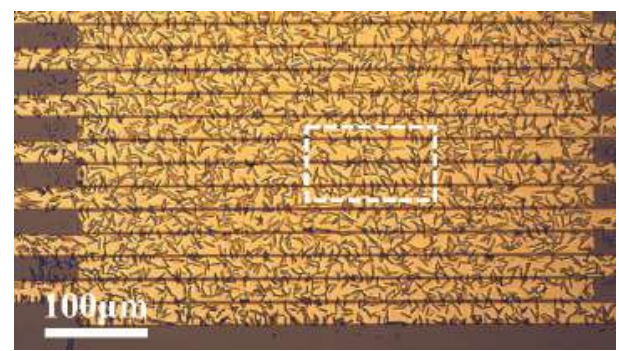

C)

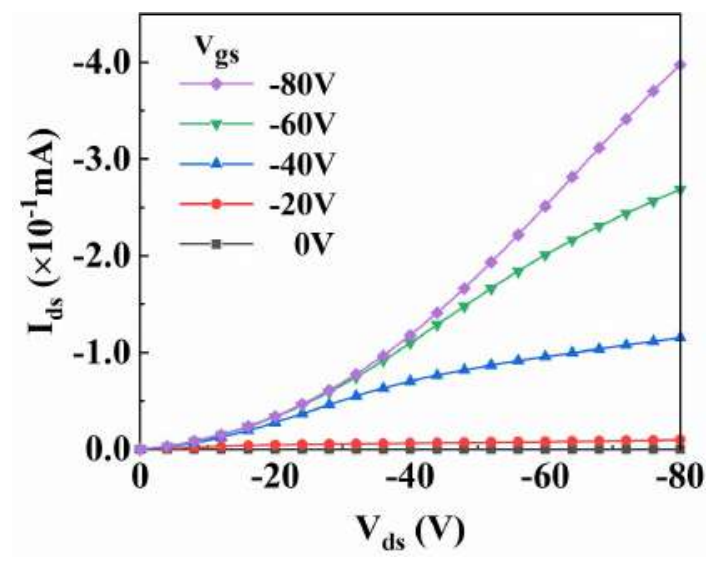

b)

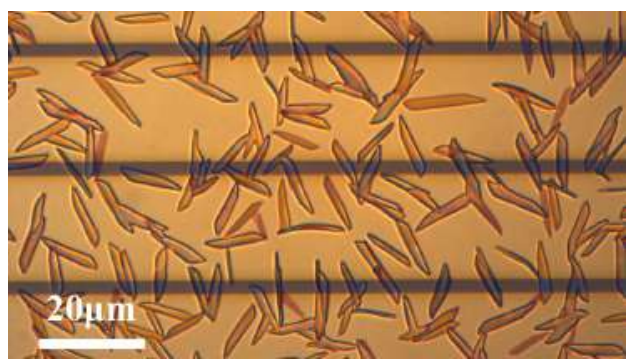

d)

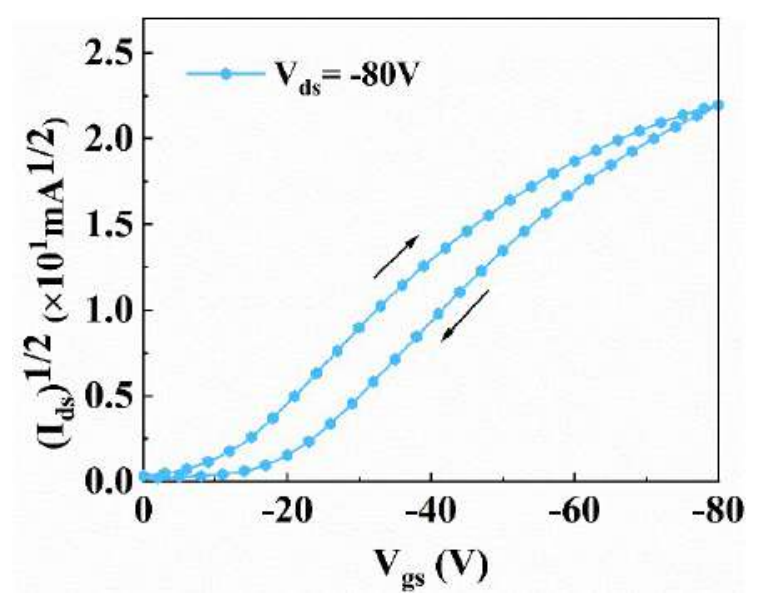

Fig. 4 (a) Optical micrograph of source and drain contacts of a bottom-contact-bottom gate transistor with TPD $\mathrm{C}_{\mathrm{C}-\mathrm{TAT}} \mathrm{T}_{\mathrm{C}}$ layer of large crystalline needles (channel length $=2.5 \mu \mathrm{m}$, channel width $=1.0 \mathrm{~cm}$ ). (b) Zoom of dashed area in (a). (c) Output characteristics of the bottom-gate-bottom-contact OFET. (d) Transfer characteristics in the saturation regime.

magnitude difference between out-of-plane and in-plane mobilities for both solid states. Interestingly, the SCLC mobility values are systematically larger than field-effect mobilities, which is rather uncommon, in particular when the molecular stacking directions are oriented parallel to the substrate. However, considering the significant impact that measurement devices generally have on the mobility data (due for instance to different contact resistances, lengths scales, electrostatic environments, carrier densities...), our results indicate that charge transport in $\mathrm{TPD}_{\mathrm{C8}}-\mathrm{TAT}_{\mathrm{C} 8}$ thin films, in both the mesophase and the crystalline state, is almost isotropic.

\section{Experimental}

Film deposition and thermal processing: Thin films were deposited on top of different substrates (i.e. $\mathrm{Si} / \mathrm{SiO}_{2}$, glass/ITO/PEDOT: PSS or $\mathrm{Si}_{3} \mathrm{~N}_{4}$ ) by spin coating an anhydrous chloroform (>99\%) solution of $\mathrm{TPD}_{\mathrm{C8}}-\mathrm{TAT}_{\mathrm{C8}}$ in $\mathrm{N}_{2}$ atmosphere. Different annealing steps were applied under nitrogen ambient to achieve desired solid states:

a) Nematic state: annealing at $180^{\circ} \mathrm{C}$ for 5 seconds (isotropic phase), followed by rapid cooling to room temperature.

b) Crystalline state (small crystals): annealing at $100{ }^{\circ} \mathrm{C}$ for 5 minutes, followed by $120{ }^{\circ} \mathrm{C}$ for 5 minutes and $145{ }^{\circ} \mathrm{C}$ for 40 minutes (cold crystallization). The gradual increase in temperature is used to avoid film de-wetting.

c) Crystalline state (large crystals): annealing at $180{ }^{\circ} \mathrm{C}$ for 5 minutes, followed by rapid cooling to room temperature and subsequent heating to $145^{\circ} \mathrm{C}$ for 40 minutes.

Standard SCLC devices: The standard device structure used for hole mobility measurements is shown in Scheme S1. The Indium Tin Oxide (ITO) coated glass substrates were first cleaned in soapsuds, deionized water, acetone, isopropyl alcohol at $45{ }^{\circ} \mathrm{C}$ for 15 minutes and treated by UV-ozone for 30 minutes. Poly (3,4- ethylenedioxythiophene): polystyrene sulfonate (PEDOT: PSS) was spin-coated on top of the ITO (5000 rpm for $60 \mathrm{~s}$ ), dried at $140{ }^{\circ} \mathrm{C}$ for 30 minutes in $\mathrm{N}_{2}$ atmosphere and used as hole injection electrode. A $20 \mathrm{mg} \cdot \mathrm{mL}^{-1}$ chloroform solution of TPD $\mathrm{C8}^{-}$ TAT $_{C 8}$ was spin-coated on top of the PEDOT:PSS layer under nitrogen ambient. Different thicknesses were achieved using different spin speeds (typically, $1500 \mathrm{rpm}$ for $60 \mathrm{~s}$ leads to a 250 $\mathrm{nm}$ thick layer). Lastly, $7 \mathrm{~nm}$ thick $\mathrm{MoO}_{3}$ and $120 \mathrm{~nm}$ thick $\mathrm{Ag}$ layers were deposited by physical vapor deposition on top of the active layer under vacuum $\left(<10^{-6} \mathrm{mbar}\right)$. The electrode surface area varied between $1.0 \mathrm{~mm}^{2}$, and $2.5 \times 10^{-2} \mathrm{~mm}^{2}$. The small device areas were necessary to avoid the compliance of the measurement equipment for high mobility samples.

Thick structure SCLC devices: Thick devices were manufactured by first structuring the ITO layers with acid etching into an array 
of parallel, $1 \mathrm{~mm}$ wide, ITO stripes. Both glass/ITO substrates were coated by PEDOT: PSS and used as building block for the final device (Scheme S2). A suitable amount of molecular powder $\left(0.5^{\sim} 3 \mathrm{mg}\right.$ ) was accurately weighted (to the nearest one-hundredth of a $\mathrm{mg}$ ) and was deposited on the center of one structured glass/ITO substrate. Several spacers $(8,20,28 \mu \mathrm{m}$ thick) were added on the four corners in order to define the approximate final active layer thickness. The second ITO-glass substrate was put on top of the molecular powder with the electrodes being perpendicularly oriented with respect to the ITO strips of the bottom substrate. The sample was then heated above the isotropic transition temperature $\left(180^{\circ} \mathrm{C}\right)$ for several minutes under mechanical compression. Being in the liquid state, the molecules were able to spread over the device area, while a mechanical pressure was constantly and evenly applied over the entire surface of the cell. After cooling to room temperature, the samples were eventually annealed again at $145{ }^{\circ} \mathrm{C}$ for 40 minutes to form the crystalline phase. The final device thickness $L$ of the active layer was estimated by taking into account the mass $m$ of the organic materials deposited on the first substrate and the area $S$ occupied by the organic materials as evaluated from the micrograph (red area of Scheme S2d, according to $L=m /(\rho \cdot S)$ and assuming a volume density $\rho$ of $1.0 \mathrm{mg} \mathrm{cm}^{-3}$. The individual diode area was fixed to $1 \mathrm{~mm}^{2}$. For each sample, approximately 4 individual diodes could be characterized.

Field-effect transistors: Bottom-contact-bottom gate transistors were fabricated using commercially available $\mathrm{Si} / \mathrm{SiO}_{2}$ substrates with lithographically defined source and drain contacts. The latter were composed of ITO $(10 \mathrm{~nm}) / \mathrm{Au}(30 \mathrm{~nm})$ bilayers defining a channel length of $2.5 \mu \mathrm{m}$ and channel width of $1.0 \mathrm{~cm}$. The $230 \mathrm{~nm}$ thick $\mathrm{SiO}_{2}$ layer was used as a gate dielectric. The substrates were cleaned in soapsuds, acetone and isopropyl alcohol and subsequently for 30 minutes in an UVozone system. A $5 \mathrm{mg} \mathrm{ml}^{-1}$ concentrated anhydrous chloroform solutions of TPD $\mathrm{CB}_{\mathrm{C}}-\mathrm{TAT}_{\mathrm{C} 8}$ were spin-coated (1500 rpm for $60 \mathrm{~s}$ ) in $\mathrm{N}_{2}$ ambient.

Top-contact-bottom gate transistors were fabricated using a silicon substrate with a $200 \mathrm{~nm}$ thermal silicon oxide $\left(\mathrm{SiO}_{2}\right)$ layer as gate dielectric. The substrates were cleaned in soapsuds, acetone and isopropyl alcohol and subsequently for 30 minutes in an UV-ozone system. A $5 \mathrm{mg} \mathrm{ml}^{-1}$ concentrated anhydrous chloroform solutions of TPD $_{\mathrm{C} 8}-\mathrm{TAT}_{\mathrm{C} 8}$ were spin-coated (1500 rpm for $60 \mathrm{~s}$ ) in nitrogen ambient. The top electrodes $(7 \mathrm{~nm}$ $\mathrm{MoO}_{3}$ and $120 \mathrm{~nm} \mathrm{Ag}$ ) were thermal evaporated on top of the active layer under secondary vacuum $\left(<10^{-6} \mathrm{mbar}\right)$.

Electrical characterization: SCLC and OFET devices were characterized in nitrogen ambient using a probe station and a Keithley 4200 semiconductor analyzer. A Peltier thermomodule was used for temperature dependent SCLC measurements. The SCLC devices were placed directly on top of the Peltier module. The temperature was measured by a RTD Pt100 sensor and varied between $263 \mathrm{~K}$ to $323 \mathrm{~K}$. The fieldeffect hole mobility was calculated by fitting the measured transfer curves in the saturation regime to the standard transistor model. ${ }^{[44]}$
Film thickness measurements: The nematic state thin film thickness was measured using a Bruker Dektak profilometer with a scan speed of $10 \mu \mathrm{m} \mathrm{s}^{-1}$, lateral resolution of $0.6 \mu \mathrm{m}$, and stylus force $1.0 \mathrm{mg}$. The crystalline state thin film thickness and roughness was measured using atomic force microscopy (AFM).

Differential scanning calorimetry (DSC): Differential scanning calorimetry (DSC) were performed with a TA Instruments Q1000 instrument, operated at a scanning rate of $5{ }^{\circ} \mathrm{C} \mathrm{min}^{-1}$ on heating and on cooling.

Grazing Incidence Wide-Angle X-ray Scattering (GIWAXS): GIWAXS measurements were conducted at PLS-II 9A U-SAXS beamline of Pohang Accelerator Laboratory (PAL) in Korea. The $X$-rays coming from the vacuum undulator (IVU) were monochromated using Si (111) double crystals and focused on the detector using K-B type mirrors. Patterns were recorded with a 2D CCD detector (Rayonix SX165). The sample-todetector distance was about $222 \mathrm{~mm}$ for energy of $11.07 \mathrm{keV}$ (1.120 ̊̊).

Microfocus X-ray diffraction: Microfocus X-ray diffraction experiments were carried out at the ID13 beamline of the European Synchrotron Radiation Facility (ESRF) in Grenoble (France). For the measurements spin-coated films of TPDC8TATC8 were crystallized on a $1 \mu \mathrm{m}$ thick suspended $\mathrm{Si}_{3} \mathrm{~N}_{4}$ window from Silson Ltd. (UK). The region of interest on a membrane was identified with an on-axis optical microscope. The X-ray diffraction measurements were performed with a photon energy of $13 \mathrm{keV}(0.9537 \AA$ ) at a sample-to-detector distance of $(155.96 \pm 0.02) \mathrm{mm}$. The footprint of the X-ray beam was $2.5 \times 2.5 \mu \mathrm{m}^{2}$. The sample was fixed on a piezo translation stage, which in turn was placed on a hexapod stage for coarse movements. The sample was 2D scanned with a step of $1 \mu \mathrm{m}$ along both coordinates. The resulting 2D diffractograms were centered, geometrically corrected and calibrated using a corundum standard.

\section{Conclusions}

In summary, we have explored the solid-state structure and hole transport in a donor-acceptor-donor type planar dumbbellshaped molecular semiconductor comprising two triazatruxene(TAT) end units and a central thiophenethienopyrroledione-thiophene (TPD) unit. The results reveal that these molecules self-assemble into either an uncommon bridged hexagonal columnar nematic liquid crystalline phase or a daughter, crystalline phase, depending on the thermal history. The $(h k O)$ peaks of the crystalline phase could be indexed to an oblique unit cell, which is equivalent to a slightly deformed hexagonal lattice, suggesting that the observed crystalline phase stems from the parent hexagonal liquid-crystalline phase. Both phases exhibit a molecular stacking direction lying in plane and oriented perpendicular to the molecular axes. The conjugated segments connecting two TAT units then act as intercolumnar conjugated bridges and are at the origin of unique charge transport properties. Charge transfer is indeed no longer limited to intermolecular hopping along onedimensional columnar stacks, as usually observed in other single TAT-derivatives or discotic molecules, but becomes 
effective in 3D. In the mesophase, the out-of-plane hole transport was estimated by SCLC to be about $10^{-3} \mathrm{~cm}^{2} \mathrm{~V}^{-1} \mathrm{~s}^{-1}$, slightly above the field-effect in-plane mobility. On the other hand, an outstandingly high out-of-plane SCLC mobility of 0.17 $\mathrm{cm}^{2} \mathrm{~V}^{-1} \mathrm{~s}^{-1}$, in combination with a low activation energy of 30 $\mathrm{meV}$, was measured in the crystalline phase. Although only a lower limit of $0.05 \mathrm{~cm}^{2} \mathrm{~V}^{-1} \mathrm{~s}^{-1}$ could be obtained so far for the inplane field-effect mobility, the results support the conclusion that the molecular assembly allows for efficient charge transport perpendicular as well as parallel to the molecular stacking direction. Overall, our observations suggest that the two highly planar TAT end-groups are effectively driving the molecules into a unique 3D conducting bridged columnar phase, wherein the molecules are bridging adjacent columns or molecular stacks through the insulating aliphatic domains. As a consequence, 3D charge-transport with a low degree of anisotropy, a feature rarely observed in solution processable crystalline small molecules, could be demonstrated.

\section{Conflicts of interest}

There are no conflicts to declare.

\section{Acknowledgements}

The authors gratefully acknowledge the C3Fab (ICube) platform for access to the equipment and Nicolas Zimmermann, as well as Sadiara Fall for technical support. The authors also thank Pohang Accelerator Laboratory (PAL) for the opportunity to perform GIWAXS measurements, MEST, and POSTECH for supporting these experiments, Dr. Hyungju Ahn for adjustment and help, and other colleagues from the 9A USAXS beamline for assistance. D.A. Ivanov and M. Rosenthal thank Manfred Burghammer for assistance with the experiment at the ID13 beamline of the European Synchrotron Radiation Facility (ESRF). The authors also gratefully acknowledge the different following funding programs. This research was supported by Leading Foreign Research Institute Recruitment Program through the National Research Foundation of Korea (NRF) funded by the Ministry of Science, ICT \& Future Planning (NRF-2010-00453). D.A. Ivanov acknowledges financial support from the Ministry of Science and Higher Education of the Russian Federation within State Contract 075-15-2019-1889. J. Jing acknowledges financial support from China Scholarship Council (CSC).

\section{References}

[1] S. Fratini, M. Nikolka, A. Salleo, G. Schweicher, H. Sirringhaus, Nat. Mater. 2020, 19, 491.

[2] S. K. Park, J. H. Kim, S. Y. Park, Adv. Mater. 2018, 30, 1704759.

[3] G. Feng, Y. Xu, J. Zhang, Z. Wang, Y. Zhou, Y. Li, Z. Wei, C. Li, W. Li, J. Mater. Chem. A 2016, 4, 6056.

[4] G. Schweicher, Y. Olivier, V. Lemaur, Y. H. Geerts, Isr. J. Chem. 2014, 54, 595.

[5] O. P. Lee, A. T. Yiu, P. M. Beaujuge, C. H. Woo, T. W. Holcombe, J. E. Millstone, J. D. Douglas, M. S. Chen, J. M. J. Fréchet, $A d v$. Mater. 2011, 23, 5359.
[6] Y. Chang, X. Zhu, K. Lu, Z. Wei, J. Mater. Chem. A 2021, 9, 3125.

[7] M. C. Scharber, N. S. Sariciftci, Prog. Polym. Sci. 2013, 38, 1929.

[8] O. Ostroverkhova, Chem. Rev. 2016, 116, 13279.

[9] H. Arora, V. Bhalla, M. Kumar, RSC Adv. 2015, 5, 32637.

[10] K. Takimiya, I. Osaka, T. Mori, M. Nakano, Acc. Chem. Res. 2014, 47, 1493.

[11] M. Sawamoto, M. J. Kang, E. Miyazaki, H. Sugino, I. Osaka, K. Takimiya, ACS Appl. Mater. Interfaces 2016, 8, 3810.

[12] H. Ebata, T. Izawa, E. Miyazaki, K. Takimiya, M. Ikeda, H. Kuwabara, T. Yui, J. Am. Chem. Soc. 2007, 129, 15732.

[13] Y. Li, R. G. Clevenger, L. Jin, K. V. Kilway, Z. Peng, J. Mater. Chem. C 2014, 2, 7180.

[14] K. Rakstys, A. Abate, M. I. Dar, P. Gao, V. Jankauskas, G. Jacopin, E. Kamarauskas, S. Kazim, S. Ahmad, M. Grätzel, M. K. Nazeeruddin, J. Am. Chem. Soc. 2015, 137, 16172.

[15] X.-C. Li, C.-Y. Wang, W.-Y. Lai, W. Huang, J. Mater. Chem. C 2016, 4, 10574.

[16] H. Zhang, Y. Liu, Y. Sun, M. Li, B. Kan, X. Ke, Q. Zhang, X. Wan, Y. Chen, Chem. Commun. 2017, 53, 451.

[17] K. Sun, Z. Xiao, S. Lu, W. Zajaczkowski, W. Pisula, E. Hanssen, J. M. White, R. M. Williamson, J. Subbiah, J. Ouyang, A. B. Holmes, W. W. H. Wong, D. J. Jones, Nat. Commun. 2015, 6, 6013.

[18] B. Kan, M. Li, Q. Zhang, F. Liu, X. Wan, Y. Wang, W. Ni, G. Long, X. Yang, H. Feng, Y. Zuo, M. Zhang, F. Huang, Y. Cao, T. P. Russell, Y. Chen, J. Am. Chem. Soc. 2015, 137, 3886.

[19] S. Fratini, S. Ciuchi, D. Mayou, G. T. de Laissardière, A. Troisi, Nat. Mater. 2017, 16, 998.

[20] Z.-F. Yao, J.-Y. Wang, J. Pei, Cryst. Growth Des. 2018, 18, 7.

[21] I. Bulut, P. Chávez, A. Mirloup, Q. Huaulmé, A. Hébraud, B. Heinrich, S. Fall, S. Méry, R. Ziessel, T. Heiser, P. Lévêque, N. Leclerc, J. Mater. Chem. C 2016, 4, 4296.

[22] S. Ya-Rui, W. Hui-Ling, S. Ya-Ting, L. Yu-Fang, CrystEngComm 2017, 19, 6008.

[23] E. M. García-Frutos, U. K. Pandey, R. Termine, A. Omenat, J. Barberá, J. L. Serrano, A. Golemme, B. Gómez-Lor, Angew. Chem. Int. Ed. 2011, 50, 7399.

[24] T. Han, I. Bulut, S. Méry, B. Heinrich, P. Lévêque, N. Leclerc, T. Heiser, J. Mater. Chem. C 2017, 5, 10794.

[25] H. lino, T. Usui, J.-i. Hanna, Nat. Commun. 2015, 6, 6828.

[26] O. Kwon, X. Cai, W. Qu, F. Liu, J. Szydłowska, E. Gorecka, M. J. Han, D. K. Yoon, S. Poppe, C. Tschierske, Adv. Funct. Mater., 2021, 31, 2102271.

[27] C. Dominguez, B. Heinrich, B. Donnio, S. Coco, P. Espinet, Chem. Eur. J., 2013, 19, 5988-5995.

[28] T. He, X. Zhang, J. Jia, Y. Li, X. Tao, Adv. Mater. 2012, 24, 2171.

[29] R. W. I. d. Boer, M. Jochemsen, T. M. Klapwijk, A. F. Morpurgo, J. Niemax, A. K. Tripathi, J. Pflaum, J. Appl. Phys. 2004, 95, 1196.

[30] J. A. Röhr, X. Shi, S. A. Haque, T. Kirchartz, J. Nelson, Phys. Rev. Appl. 2018, 9, 044017.

[31] G. A. H. Wetzelaer, P. W. M. Blom, Phys. Rev. B 2014, 89, 241201.

[32] R. J. Bushby, S. M. Kelly, M. O'Neill, Liquid Crystalline Semiconductors: Materials, properties and applications, Springer Netherlands, Dordrecht 2013, 39.

[33] J. A. Röhr, R. C. I. MacKenzie, J. Appl. Phys. 2020, 128, 165701.

[34] M. Bajpai, K. Kumari, R. Srivastava, M. N. Kamalasanan, R. S. Tiwari, S. Chand, Solid State Commun 2010, 150, 581.

[35] P. W. M. Blom, M. J. M. de Jong, M. G. van Munster, Phys. Rev. B 1997, 55, R656.

[36] J. M. Montero, J. Bisquert, J. Appl. Phys. 2011, 110, 043705.

[37] C.-Y. Liu, S.-A. Chen, Macromol. Rapid Commun. Mass Spectrom. 2007, 28, 1743.

[38] C. Li, L. Duan, H. Li, Y. Qiu, J. Phys. Chem. C 2014, 118, 10651.

[39] X. Bai, K. Zong, J. Ly, J. S. Mehta, M. Hand, K. Molnar, S. Lee, B. Kahr, J. M. Mativetsky, A. Briseno, S. S. Lee, Chem. Mater. 2017, 29, 7571.

[40] A. Benito-Hernández, U. K. Pandey, E. Cavero, R. Termine, E. M. García-Frutos, J. L. Serrano, A. Golemme, B. Gómez-Lor, Chem. Mater. 2013, 25, 117. 
[41] C. Zhao, C. Li, L. Duan, Phys. Chem. Chem. Phys. 2019, 21, 9905.

[42] V. Coropceanu, J. Cornil, D. A. da Silva Filho, Y. Olivier, R. Silbey, J.-L. Brédas, Chem. Rev. 2007, 107, 926.

[43] S. Züfle, S. Altazin, A. Hofmann, L. Jäger, M. T. Neukom, W. Brütting, B. Ruhstaller, J. Appl. Phys. 2017, 122, 115502.

[44] I. Bulut, P. Lévêque, B. Heinrich, T. Heiser, R. Bechara, N Zimmermann, S. Méry, R. Ziessel, N. Leclerc, J. Mater. Chem. A 2015, 3, 6620 . 